\title{
A fast sonochemical approach for the synthesis of solution processable $\mathrm{ZnO}$ rods
}

\author{
Marco Palumbo, ${ }^{\text {a) }}$ S. J. Henley, T. Lutz, V. Stolojan, and S. R. P. Silva \\ Nano-Electronics Centre, Advanced Technology Institute, University of Surrey, Guilford, GU2 7XH, United \\ Kingdom
}

(Received 9 January 2008; accepted 13 August 2008; published online 2 October 2008)

\begin{abstract}
A solution based sonochemical synthesis method for $\mathrm{ZnO}$ rods is presented with a resulting growth rate in excess of 15 times faster than previously reported. Such material is solution processable and could be exploited in the fabrication of transparent conductors and/or large area electronics via inkjet printing methods or solution based self-assembly techniques. To understand the crystal structure and defects chemistry, the as-synthesized wurtzite crystal structures were compared and contrasted with rods grown by the more traditional and well characterized hydrothermal growth method. Fluorescence spectra were recorded and the emission characteristics correlated with the structural and conductive properties of the $\mathrm{ZnO}$ rods. In particular, the sonochemical crystals appear to have a higher degree of order with fewer defects. This study represents a first step toward the tailoring of the electronic properties of $\mathrm{ZnO}$ rods. In particular, we will concentrate on the influence that native defects have on electrical conduction and on photoluminescence. Furthermore, we show how the intensity of the ultrasonic power exploited in this synthesis has a direct influence on the crystal quality as revealed by a comparative study. An optimum value between $30 \%$ and $35 \%$ of the maximum amplitude of a $20 \mathrm{kHz}$ ultrasonic probe was found to give the best conditions for the growth of crystals with fewer defects density, while at ca. $25 \%$ of the maximum amplitude we observed the higher intensities for the fluorescence spectra both in the ultraviolet and in the visible range. (C) 2008 American Institute of Physics. [DOI: 10.1063/1.2988295]
\end{abstract}

\section{INTRODUCTION}

$\mathrm{ZnO}$ structures and thin films have regained increasing popularity in recent years as a result of their wide range of optical, piezoelectric, and magnetic properties. The large bandgap of $3.37 \mathrm{eV}$ and its large exciton binding energy of $60 \mathrm{meV}$ make it a very promising candidate for lasing based on excitation recombination at room temperature. ${ }^{1}$ Furthermore, $\mathrm{ZnO}$ nanostructures have found application as light emitting devices, ${ }^{2}$ solar cells, ${ }^{3} \mathrm{UV}$ photo sensors, ${ }^{4}$ piezoelectric nanogenerators, ${ }^{5}$ and gas sensors. ${ }^{6}$

The synthesis methods of $\mathrm{ZnO}$ are quite diverse including pulsed laser ablation, ${ }^{7,8}$ chemical vapor deposition (CVD), metal-organic CVD, electrodeposition. and hydrothermal solution growth. ${ }^{9,10}$ This paper proposes an inexpensive method to generate, on a large scale and at moderate temperature, $\mathrm{ZnO}$ thin films and nanostructures with controlled complexity. In a hydrothermal decomposition reaction, a 1:1 molar solution of zinc nitrate, $\mathrm{Zn}\left(\mathrm{NO}_{3}\right)_{2} \cdot 6 \mathrm{H}_{2} \mathrm{O}$ and hexamethylenetetramine (HMTA), $\left(\mathrm{CH}_{2}\right)_{6} \mathrm{~N}_{4}$ is placed in an oven at $90{ }^{\circ} \mathrm{C}$ for $6 \mathrm{~h}$. Consequently, hydroxide ions are formed by decomposition of HMTA and they react with $\mathrm{Zn}^{2+}$ to form $\mathrm{ZnO}$

$$
\begin{aligned}
& \left(\mathrm{CH}_{2}\right)_{6} \mathrm{~N}_{4}+6 \mathrm{H}_{2} \mathrm{O} \leftrightarrows 6 \mathrm{HCHO}+4 \mathrm{NH}_{3}, \\
& \mathrm{NH}_{3}+\mathrm{H}_{2} \mathrm{O} \leftrightarrows \mathrm{NH}_{4}^{+}+\mathrm{OH}^{-},
\end{aligned}
$$

\footnotetext{
a)Electronic mail: m.palumbo@surrey.ac.uk., Tel.: +44 (0) 1483686088.
}

$$
2 \mathrm{OH}^{-}+\mathrm{Zn}^{2+} \rightarrow \mathrm{ZnO}(s)+\mathrm{H}_{2} \mathrm{O} .
$$

Normally, $\mathrm{ZnO}$ rods have hexagonal polar facets, ${ }^{10}$ which are an important element for lasing applications of $\mathrm{ZnO}$ structures, as the polar facets at the extremities act as the mirrors of a Fabry-Pérot cavity, while a reduced lateral scattering is guaranteed by the high refractive index of the $\mathrm{ZnO}$ ( $n$ $=2.45$ ), with the rod effectively acting as a waveguide. ${ }^{1,11} \mathrm{An}$ imperfect 1:1 molar ratio of the starting solution may result in an acicular shape of the rods. Previously, ultrasonication of a zinc nitrate and HMTA solution has been shown to accelerate the synthesis. ${ }^{12,13}$ In this work, we prove that it is possible to enhance even further the growth rate of the $\mathrm{ZnO}$ rods by combining ultrasonication with the use of a thermal bath. Through ultrasounds, mechanical energy is transferred to a reacting medium. Part of this energy will produce heat while another will cause cavitation, i.e., the production of microbubbles in a liquid when a large negative pressure is applied. ${ }^{14}$ These microbubbles act as microreactor because of the local increase in temperature and pressure at the moment of their collapse. Of particular interest for the formation of $\mathrm{ZnO}$ structures is the production of oxygen gas and hydrogen peroxide during the sonolysis of water in an air atmosphere. ${ }^{13,14}$ This extra oxygen may also react with the zinc ions in solution to produce $\mathrm{ZnO}$

$$
2 \mathrm{O}_{2}^{-}+\mathrm{Zn}^{2+} \stackrel{))}{\rightarrow} \mathrm{ZnO}(s)+\frac{3}{2} \mathrm{O}_{2}
$$

where the symbol ))) indicates sonication (note that in a sonochemical process Eq. (3) as well is driven by sonication). 


\section{EXPERIMENTAL}

To obtain an accelerated growth of $\mathrm{ZnO}$ rods, the use of a thermal bath is essential. In most cases, we used a simple beaker full of de-ionized water at a temperature of $70{ }^{\circ} \mathrm{C}$. A thin bodied sonotrode connected to a $750 \mathrm{~W}, 20 \mathrm{kHz}$ ultrasonic processor was introduced inside the reacting solution, previously heated up to $70{ }^{\circ} \mathrm{C}$ and contained in a glass vial placed inside the thermal bath, and ultrasonic power applied with an amplitude value set varying between $20 \%$ and $40 \%$ of the maximum value. Thin bodied sonotrodes are very effective to generate a small but very intense cavitation zone, while larger sonotrodes will produce larger but less intense cavitation zones despite transmitting a bigger ultrasound power. The reacting solution consisted of a 1:1 molar mixture of $\mathrm{Zn}\left(\mathrm{NO}_{3}\right)_{2} \cdot 6 \mathrm{H}_{2} \mathrm{O}$ and $\left(\mathrm{CH}_{2}\right)_{6} \mathrm{~N}_{4}$ prepared in a range of concentrations going from $10^{-2} \mathrm{M}$ to $10^{-4} \mathrm{M}$. The recorded value of the energy transferred by the tip to the solution during the process varied between 350 and $5000 \mathrm{~J}$, directly proportional to the set amplitude value. Typically, the temperature of the external bath would drop from 70 to $50 / 55{ }^{\circ} \mathrm{C}$ at the end of the process. We found the drop in temperature to have an inverse relation with the power transferred to the solution. The maximum value and the pulse settings were $5 \mathrm{~s}$ on and $5 \mathrm{~s}$ off. In the first five seconds, energy is transferred from the microtip to the solution, while in the following $5 \mathrm{~s}$ sequential growth of the zinc oxide takes place. The produced $\mathrm{ZnO}$ rods were then separated from excess reagents and secondary reaction products via ultracentrifugation. Rods were resuspended in ultrapure water or organic solvent via sonication and vigorous agitation. Dimensions and crystal structures were determined by scanning and transmission electron microscopy (SEM and TEM). Fluorescence spectra were recorded using a xenon lamp spectrophotometer.

\section{RESULTS AND DISCUSSIONS}

The growth of $\mathrm{ZnO}$ rods was found to be dependent on using a thermal bath, which was fundamental to accelerating the growth. When a cooling bath was used or when the temperature of the solution in the vials was left to drift, we could not observe the formation of $\mathrm{ZnO}$ rods even when the sample was ultrasonicated for over $1 \mathrm{~h}$. No $\mathrm{ZnO}$ rods were formed when the thin bodied sonotrode was replaced by a larger ultrasound probe with the amplitude set at $A=70 \%$ with no external heat source applied to the reacting bath. Figure 1(a) shows that $\mathrm{ZnO}$ rods are consistently formed by two subunits attached end-to-end. In particular, the rods shown in Fig. 1(a) were synthesized from a mixture $10^{-3} \mathrm{M}$ of zinc nitrate and HMTA. As a reference, $\mathrm{ZnO}$ rods grown using the hydrothermal method starting from a $10^{-3} \mathrm{M}$ solution are shown in Fig. 1(b). The acicular shape is thought to derive from an imperfect 1:1 stoichiometry. From the analysis of similar SEM images, we observe that these rods come in two slightly different variations: the first one has two identical subunits with the same width and length, while for the second variant the two subunits have different widths but maintain consistent length. This observation on the length of the subunits appears to indicate that the growth process starts from a seed at the

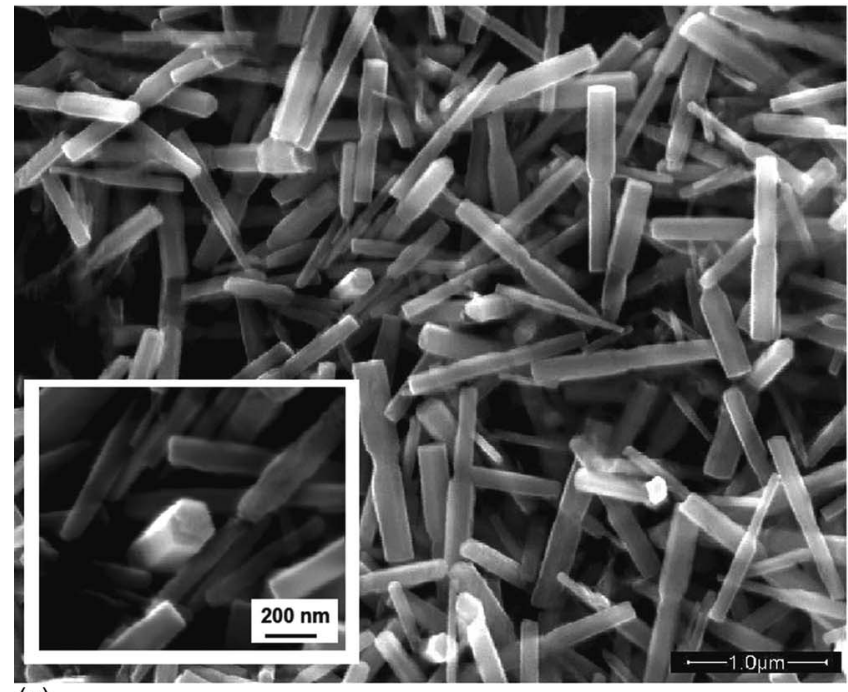

(a)

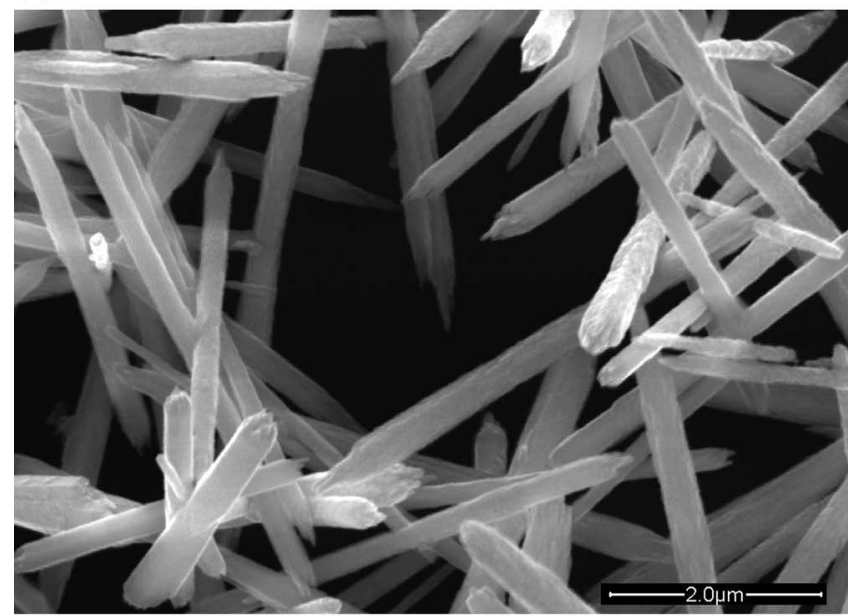

(b)

FIG. 1. (a) SEM images of the result of ultrasound treatment of a $10^{-3} \mathrm{M}$ zinc nitrate and hexamethyletetramine solution. In the inset in the bottomleft corner, a detail of a flat hexagonal rod end is shown. (b) Hydrothermally synthesized $\mathrm{ZnO}$ rods.

center of the rods and then proceeds at constant speed in opposite directions along the same axis. Assuming a total rod length of $1.3 \mu \mathrm{m}$ as for the rods in Fig. 1(a), the growth rate is $7800 \mathrm{~nm} \mathrm{~h}^{-1}$, over 15 times faster than previously reported growth rates for sonochemical synthesis. ${ }^{13}$ Theoretically, our method could also be exploited for the growth of $\mathrm{ZnO}$ wires, provided that a steady flow of reagents is supplied to the reaction zone. This is at present the object of ongoing experimentation. A close examination of the $\mathrm{ZnO}$ rods reveals a hexagonal cross section as shown in the inset of Fig. 1(a), a feature relevant to lasing applications of structured $\mathrm{ZnO}$ films. ${ }^{1,11}$ The aspect ratio, length/width of the rods can be tuned by varying the molar concentration of the initial mixture. On average, from a $10^{-2} \mathrm{M}$ solution rods with a width of ca. $400 \mathrm{~nm}$ and lengths of approximately $1 \mu \mathrm{m}$ were obtained. It is worth stating that rods synthesized by a $10^{-2} \mathrm{M}$ solution have a tendency to be thicker in the center and thinner at the extremities. Therefore, the value taken into account for the width was the average of the width measured halfway down the length of the two subunits. This particular phenomenon could be explained with the decrease in the free 


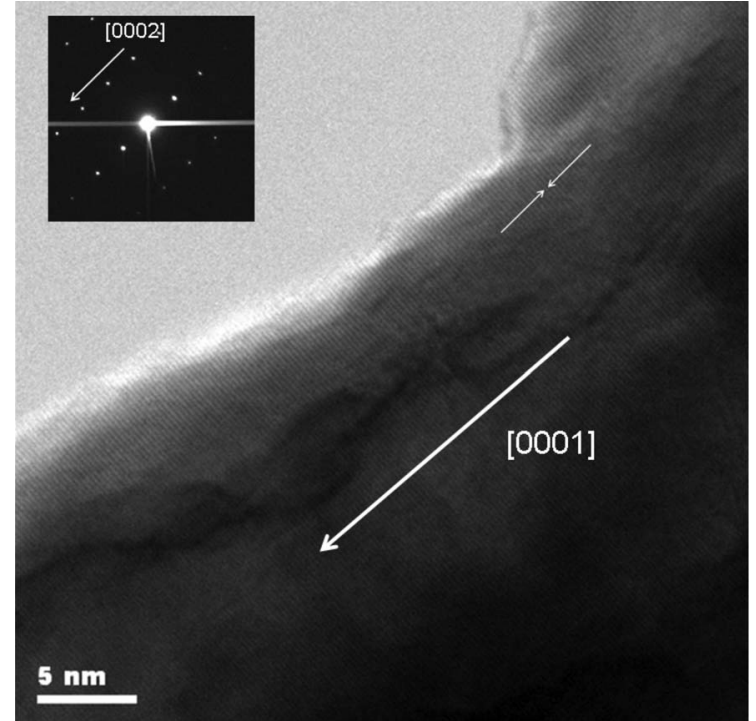

FIG. 2. Crystallographic planes as revealed from high resolution TEM analysis. The distance between two adjacent planes indicated by arrows is $0.26 \mathrm{~nm}$. In the inset (a), the corresponding electron diffraction pattern is shown.

zinc concentration during the growth process, which causes the variation in the ratio between the two-dimensional and the one-dimensional growth mode discussed in this paper. ${ }^{15}$ From a $10^{-3} \mathrm{M}$ solution, we obtained $\mathrm{ZnO}$ rods with a width of approximately $200 \mathrm{~nm}$ and length of $1.3 \mu \mathrm{m}$, giving a resulting aspect ratio of 6.5 . Slight variations in the growth parameters at times result in wider and longer rods (e.g., 500 $\mathrm{nm}$ wide and $3.5 / 4 \mu \mathrm{m}$ long) with a consistent aspect ratio. This class of rods resulted in more uniform width values along their growth axis, in contrast with those prepared from the $10^{-2} M$ mixtures. Again, this seems to confirm that at low zinc concentrations, one-dimensional growth dominates over the two-dimensional growth mode.

To obtain the crystal structure of the $\mathrm{ZnO}$ rods produced, a few drops of a diluted dispersion in isopropanol were cast on a TEM grid coated with amorphous carbon. Due to rod thickness, lattice fringes were only visible at the very edges of the rods, as shown in Fig. 2. The high resolution TEM analysis indicates a wurzite crystallographic structure with the growth along the [0001] direction, the polar $c$-axis of the $\mathrm{ZnO}$ crystal lattice. The distance between the (0002) planes indicated by the arrows in Fig. 2 is of $0.26 \mathrm{~nm}$, a value consistent with the wurzite crystal structure. The relative open structure of such a crystal with a hexagonal closed packed lattice, where the $\mathrm{Zn}$ atoms occupy half of the tetrahedral sites and where all the octahedral sites are empty, leaves plenty of sites to accommodate intrinsic defects and extrinsic dopants. ${ }^{9}$ This allows scope to fully exploit the properties of the $\mathrm{ZnO}$ structures by tuning its properties through doping (e.g., dilute magnetic semiconductor applications $^{16-18}$ ). The control of its defects chemistry and of the associated charge carriers would be ideally favored by such open structures.

The luminescent properties of the $\mathrm{ZnO}$ rods at room temperature were investigated using a xenon lamp fluorescence spectrophotometer. A monochromator and filter were

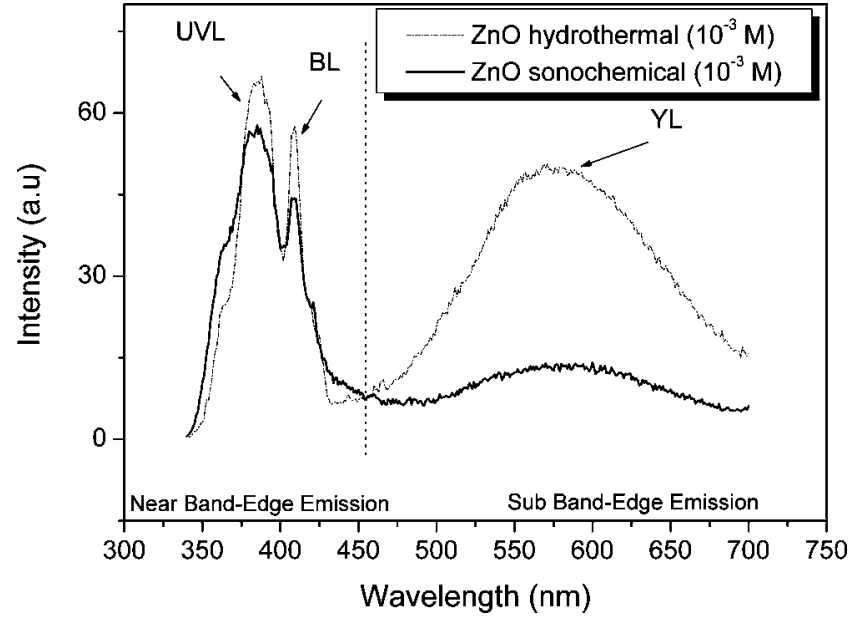

FIG. 3. Fluorescence intensity vs emission wavelength for $\mathrm{ZnO}$ rods grown by the sonochemical method vs the fluorescence intensity of $\mathrm{ZnO}$ rods synthesized by hydrothermal decomposition.

used to isolate the excitation wavelength of $300 \mathrm{~nm}$. Fluorescence spectra were recorded both for $\mathrm{ZnO}$ rods grown by the sonochemical method and for those grown by hydrothermal decomposition. In both cases the concentration of the initial reactants was $10^{-3} \mathrm{M}$. To evaluate the effect of selfabsorbance, we used a UV-Vis spectrometer scanning the response of the samples over a wavelength range going from 300 to $800 \mathrm{~nm}$. At the concentrations investigated and for the purpose of our investigation, the UV-Vis response indicated a very negligible self-absorbent behavior (i.e., no change in relative peak intensities when the concentration is varied). As indicated in Fig. 3 by the dashed line, two separate band emissions are evident for both samples. The first band emission encountered (at high energy levels) is the near-band gap emission (NBE) and in our case presents a double peak at 385 and $409 \mathrm{~nm}$ for the sonochemical sample, while the hydrothermal $\mathrm{ZnO}$ rods NBE peak positions are at 388 and $409 \mathrm{~nm}$. The first of the two peaks is actually in the ultraviolet luminescence (UVL), while the second one represents the blue luminescence (BL). The UVL for the sonochemical rods has a full width at half maximum (FWHM) of 43 nm, i.e., a $\Delta E$ of approximately $0.35 \mathrm{eV}$, while for the hydrothermal rods the FWHM of the UVL is of $34 \mathrm{~nm}$, i.e., a $\Delta E$ of approximately $0.28 \mathrm{eV}$. The BL peak has a FWHM of $23 \mathrm{~nm}$, i.e., a $\Delta E$ of approximately $0.16 \mathrm{eV}$, for the sonochemical $\mathrm{ZnO}$, while the BL FWHM for the hydrothermal $\mathrm{ZnO}$ is 20 $\mathrm{nm}$, i.e., a $\Delta E$ of $0.14 \mathrm{eV}$. As it can be appreciated, both UVL and $\mathrm{BL}$ peaks are slightly narrower in the hydrothermal $\mathrm{ZnO}$ rods case. The band gap energy of $\mathrm{ZnO}$ is $3.37 \mathrm{eV}$, corresponding to $360 \mathrm{~nm}$. The second band emission is the socalled subband gap emission (SBE). The emission maximum is at $587 \mathrm{~nm}$ for the sonochemical $\mathrm{ZnO}$ and $569 \mathrm{~nm}$ for the hydrothermal rods. In this case the half maximum width is of $119 \mathrm{~nm}$, i.e., a $\Delta E$ of $0.43 \mathrm{eV}$ for the sonochemical sample and $131 \mathrm{~nm}$, i.e., a $\Delta E$ of 0.46 , for the hydrothermal rods. This time, the latter sample shows a broader peak than the sonochemical $\mathrm{ZnO}$ rods. It has been shown that the width of the visible peak, at least for yellow-redshifted peaks, can be directly associated with inhomogeneities within single rods rather than morphology discrepancies among different 


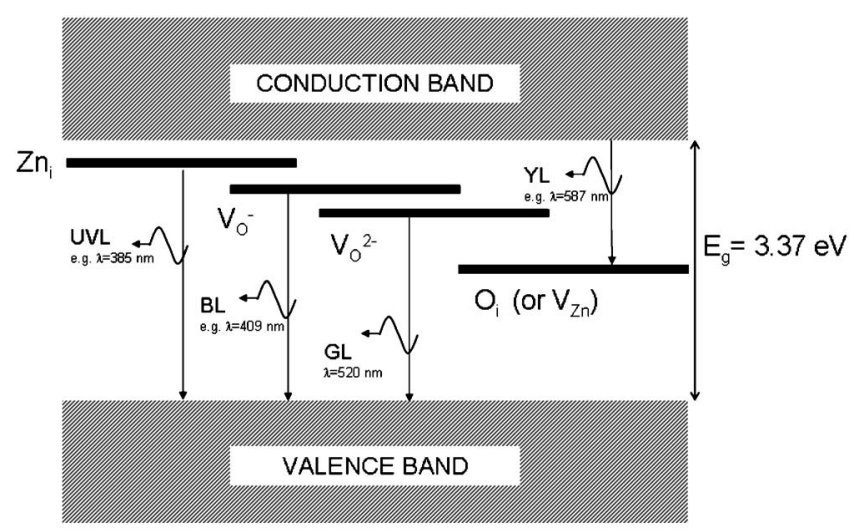

FIG. 4. A simplified $\mathrm{ZnO}$ (sonochemical) band gap showing the different emission associated with various defects. As example, the wavelengths of the sonochemical $\mathrm{ZnO}$ sample are indicated.

rods. ${ }^{19}$ Comparing the value calculated for the SBE peaks in our case, with values reported in literature for solutiongrown $\mathrm{ZnO}$ rods, ${ }^{19}$ these relatively narrow ranges seem to indicate that the rods are structurally homogeneous. The attribution of these bands (NBE and SBE) to the intrinsic defects of the undoped $\mathrm{ZnO}$ gives us an interesting insight into the rich defect chemistry of this oxide.

A thorough understanding of the correlation between defects and their associate charge carriers with the material properties is necessary to achieve successful applications of $\mathrm{ZnO}$ structures. Although $\mathrm{ZnO}$ defects have been studied in the past, ${ }^{20}$ a similar approach is still largely lacking for the novel structures available today. ${ }^{9}$ Native defects in undoped $\mathrm{ZnO}$ are donorlike in nature and, therefore, the material has $n$-type conductor characteristics. A particular aspect that needs also to be taken in consideration is that defects might be aliovalent (i.e., they have different ionization degrees). For $\mathrm{ZnO}$, the donor defects are due to interstitial zinc and oxygen vacancies. Accordingly the well-known KrögerVinch notation, ${ }^{21}$ we will hereafter refer to them as $\mathrm{Zn}_{i}$ and $V_{\mathrm{O}}$, respectively. Because both types of defect tend to donate two electrons each, it is difficult to distinguish their contribution through electrical measurements. However, it has been shown that it is possible to correlate defect spectral features with properties such as conductivity and, therefore, discriminate the contribution of one defect from the others. ${ }^{22}$ In Fig. 4, a simplified and general model of the band gap of the $\mathrm{ZnO}$ is shown. Only in the case of the $V_{\mathrm{O}}$, two different degrees of ionization have been considered (charge and number of charges are indicated by the subscripted terms). In addition to the already stated donor defects $\mathrm{Zn}_{i}$ and $V_{\mathrm{O}}$, the acceptorlike defects $\mathrm{O}_{i}$ (interstitial oxygen) and $V_{\mathrm{Zn}}$ (zinc vacancy) are also taken into account. For the sake of space they fictionally share the same energy level although it should be clear that they do have in reality separate levels. As illustrated in Fig. 4, the UVL is due to an energetic transition from the level occupied by $\mathrm{Zn}_{i}$ to the valence band, while the BL can be attributed to an energy exchange between the $V_{\mathrm{O}}^{-}$and the valence band. We arrive at this conclusion by carefully comparing the spectral features given in Fig. 3 with known $\mathrm{ZnO}$ band gaps discussed in the literature. ${ }^{9,20}$ Although some discrepancies in the absolute positions of the defect energy levels were observed, the relative position of the defect levels is consistent with those previously reported. The attribution of the green luminescence (GL) to the oxygen vacancy is widely accepted, although the contribution of the bulk defects versus the defects on the $\mathrm{ZnO}$ surface is still debated. ${ }^{12,19}$ We attribute GL to the transition occurring between the energy level occupied by $V_{\mathrm{O}}^{2-}$ and the valence band. As it can be observed from Fig. 3 , neither the sonochemical nor the hydrothermal $\mathrm{ZnO}$ rod spectra present a well-defined green peak and this is somehow to be expected at room temperature. ${ }^{23}$ Instead, the subband gap is spread over a relative large range (from 450 to $700 \mathrm{~nm}$ approximately). The peak itself of this band is shifted toward the yellow region at $587 \mathrm{~nm}$ for the sonochemical $\mathrm{ZnO}$ rods and $569 \mathrm{~nm}$ for the hydrothermal sample. Yellow luminescence (YL) has been reported to be temperature dependent according to the equation $E_{e A}=E_{\text {gap }}$ $-E_{A}+0.5 k T$, where $k$ is the Boltzmann constant, $T$ is the temperature, $E_{\text {gap }}$ is the band gap energy, and $E_{A}$ is the acceptor binding energy. ${ }^{22}$ The calculated $E_{A}$ is in our case of $1.345 \mathrm{eV}$ for the sonochemical sample and $1.275 \mathrm{eV}$ for the hydrothermal $\mathrm{ZnO}$ rods. An increase in YL intensity indicates an increase in the acceptorlike defects [i.e., an increase in interstitial oxygen $\mathrm{O}_{i}$ and/or zinc vacancy $V_{\mathrm{Zn}}$ (Ref. 22)] that will compensate the effect of the native $n$-type doping and consequently will affect the conductivity of the $\mathrm{ZnO}$ structure. Although, in Fig. 4, a transition between the conduction band and the $\mathrm{O}_{i}$ or $V_{\mathrm{Zn}}$ (or both) is indicated, interband transitions between donor defects and acceptor defects cannot at this stage be ruled out (in this case the energy level associated with $\mathrm{O}_{i}$ or $V_{\mathrm{Zn}}$ in Fig. 4 should be shifted toward the valence band). The influence of acceptor-like defects turns out to be very relevant in our case because from Fig. 3, we note that the intensity of the YL peak is much higher for the hydrothermal $\mathrm{ZnO}$ than for the sonochemical sample (we also notice a decrease in the intensity for UVL and BL peaks as well as for sonochemical $\mathrm{ZnO}$ rods). This seems to indicate that sonochemical $\mathrm{ZnO}$ rods are crystals with fewer defects in comparison with the hydrothermal sample. One of the best indications of the quality of the crystals comes from the analysis of the intensity ratio between the sum of GL and $\mathrm{BL}$ (the green component of the sub-band-edge emission) and the UVL intensity $[(\mathrm{BL}+\mathrm{GL}) / \mathrm{UVL}]$. An increase in this ratio indicates a decrease in the resistivity of $\mathrm{ZnO}^{22} \mathrm{Al}-$ though no clear GL peak can be recognized, there is undoubtedly a green component in the SBE. Taking into account the intensity at $520 \mathrm{~nm}$ for both spectra, the ratio goes from 0.93 for the sonochemical rods to 1.31 for the hydrothermal $\mathrm{ZnO}$. This, in agreement with the previous discussions on the intensity of YL, indicates that the sonochemical method is likely to produce crystals of higher quality. The fluorescence data show that the nature and density of the defects are different, and this in turn could influence the future doping strategies of the $\mathrm{ZnO}$ rods. Although further research is necessary to understand this phenomenon-and we are already envisaging, for example, a photoluminescence study as a function of temperature-we already notice that in the sonochemical method, the sonolysis of water in oxygen gas and hydrogen peroxide creates an oxygen rich 

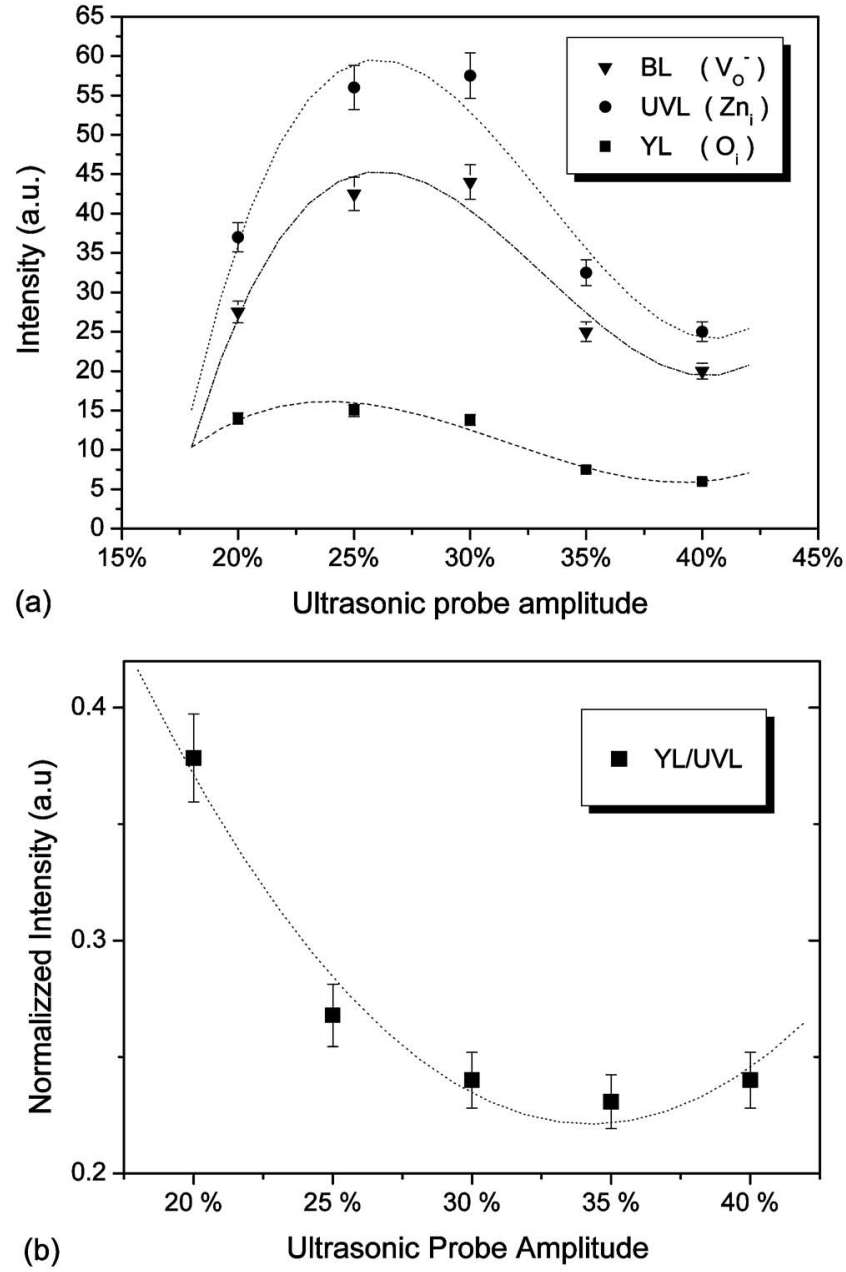

FIG. 5. (a) Variation in the sonochemical $\mathrm{ZnO}$ rod peak intensity as a function of the sonoprobe amplitude. The fitted curves are shown as guide to the eye (dotted lines for the UVL peak and dashed-dot and dashed for BL and YL peaks, respectively). (b) Normalized YL/UVL intensity-taking into account yield discrepancies- vs the ultrasonic probe amplitude. A fitted curve is shown as guide for the eyes, clearly showing an optimum minimum.

environment. This extra oxygen should also be taken into consideration in the formation of the $\mathrm{ZnO}$ crystals. This and other differences in the number and volumes of elements involved in the crystal formation or in growth parameters, such as local temperature and pressure, are likely candidates to explain the differences in crystal structure obtained by the two growth methods.

In an attempt to understand the influence of the ultrasonic power transferred to the reacting solution over the crystal quality, we undertook a systematic study in which consistent volumes and concentrations $\left(15 \mathrm{ml}, 10^{-3} \mathrm{M}\right)$ of zinc nitrate and HMTA were exposed to a range of ultrasonic powers. The microsonoprobe amplitude was varied between $20 \%$ and $40 \%$ of the maximum value. Synthesized $\mathrm{ZnO}$ rods were then allowed to precipitate due to gravity over $24 \mathrm{~h}$, washed in ultrapure water, then centrifuged and resuspended in isopropanol. Finally, fluorescence spectra for each sample were recorded. The final solutions are stable over several hours and are easily redispersed by brief ultrasonification. In Fig. 5(a) a summary of the results is presented. The absolute intensity of the three major peaks-UVL, BL, and YL-has been plotted against the sonoprobe amplitude, expressed as percentage of the maximum value. To normalize the results obtained against variations in the synthesis yield, we plotted the ratio YL/UVL against the sonoprobe amplitude. As shown in Fig. 5(b), a fitted minimum is found at around $35 \%$. This indicates that around this value the quality of the crystal reaches a relative optimum. This is likely to be associated with factors like an increase in the heat transferred to the reacting solution. A further increase in the ultrasound power is likely to affect the morphology of the rods damaging the outer surface of the freshly formed $\mathrm{ZnO}$ rods. Through SEM studies, it was possible to observe that the dimensions of the rods tend to be more homogeneous when an amplitude of $20 \%-30 \%$ is used. At $35 \%$ we start to notice occasional larger rods, while at $40 \%$ there is quite a large variation in sizes and morphologies, with almost all of them presenting an irregular outer surface.

\section{CONCLUSIONS}

In conclusion, we have shown that a fast sonochemical synthesis of wurtzite $\mathrm{ZnO}$ rods, over 15 times faster than previously reported, can be achieved by placing the vial containing the reacting solution in a simple thermal bath to control the temperature drop in a preheated zinc nitrate and HMTA solution. By comparing the luminescence spectra of these rods with that of $\mathrm{ZnO}$ structures synthesized by the more established (and time-consuming) hydrothermal method, we have shown that in the sonochemical sample the crystal quality is higher perhaps due to an oxygen rich environment. The presence of a higher number of acceptorlike defects in the hydrothermal $\mathrm{ZnO}$ rods is likely to have a direct influence on rod electronic properties. We also prove that the intensity of the ultrasonic power transferred to the reacting medium has a marked influence on the crystal quality. Both methods are inexpensive routes to scale up the generation of $\mathrm{ZnO}$ thin films and structures at moderate temperatures with controlled complexity. This study has given an insight into the defects chemistry associated with these two aqueous-solution growth methods. A better understanding of the crystal defects and their influence on $\mathrm{ZnO}$ rod properties is essential for achieving successful applications of these materials.

\section{ACKNOWLEDGMENTS}

The authors wish to thank the EPSRC, U. K. for funding via a Portfolio Partnership Award.

${ }^{1}$ M. H. Huang, S. Mao, H. Feick, H. Yan, Y. Wu, H. Kind, E. Weber, R. Russo, and P. Yang, Science 292, 1897 (2001).

${ }^{2}$ J. Bao, M. A. Zimmler, F. Capasso, X. Wang, and Z. F. Ren, Nano Lett. 6, 1719 (2006).

${ }^{3}$ A. M. Peiró, P. Ravirajan, K. Govender, D. S. Boyle, P. O’Brien, D. D. C. Bradley, J. Nelson, and J. R. Durrant, J. Mater. Chem. 16, 2088 (2006).

${ }^{4}$ J. Suehiro, N. Nakagawa, S. Hidaka, M. Ueda, K. Imasaka, M. Higashihata, T. Okada, and M. Hara, Nanotechnology 17, 2567 (2006).

${ }^{5}$ J. H. Song, J. Zhou, and Z. L. Wang, Nano Lett. 6, 1656 (2006).

${ }^{6}$ J. X. Wang, X. W. Sun, Y. Yang, H. Huang, Y. C. Lee, O. K. Tan, and L. Vayssieres, Nanotechnology 17, 4995 (2006).

${ }^{7}$ S. J. Henley, M. N. R. Ashfold, and D. Cherns, Thin Solid Films 422, 69 (2002). 
${ }^{8}$ S. J. Henley, M. N. R. Ashfold, D. P. Nicholls, P. Wheatley, and D. Cherns, Appl. Phys. A: Mater. Sci. Process. 79, 1169 (2004).

${ }^{9}$ L. S. Schmidt-Mende and J. L. MacManus-Driscoll, Mater. Today 10, 40 (2007).

${ }^{10}$ L. Vayssieres, Adv. Mater. (Weinheim, Ger.) 15, 464 (2003).

${ }^{11}$ K. Govender, D. S. Boyle, P. O'Brien, D. Binks, D. West, and D. Coleman, Adv. Mater. (Weinheim, Ger.) 14, 1221 (2002).

${ }^{12}$ X. L. Hu, Y. J. Zhu, and S. W. Wing, Mater. Chem. Phys. 88, 421 (2004).

${ }^{13}$ S. H. Jung, E. Oh, K. H. Lee, W. Park, and S. H. Jeong, Adv. Mater. (Weinheim, Ger.) 19, 749 (2007).

${ }^{14}$ T. J. Mason, Practical Sonochemistry (Ellis Horwood, Chichester, 1991), p. 186.

${ }^{15}$ R. T. R. Kumar, E. McGlynn, C. McLoughlin, S. Chakrabarti, R. C. Smith, J. D. Carey, J. P. Mosnier, and M. O. Henry, Nanotechnology 18, 6 (2007).

${ }^{16}$ D. A. Schwartz, K. R. Kittilstved, and D. R. Gamelin, Appl. Phys. Lett.
85, 1395 (2004).

${ }^{17}$ N. S. Norberg, K. R. Kittilstved, J. E. Amonette, R. K. Kukkadapu, D. A. Schwartz, and D. R. Gamelin, J. Am. Chem. Soc. 126, 9387 (2004).

${ }^{18}$ D. A. Schwartz, N. S. Norberg, Q. P. Nguyen, J. M. Parker, and D. R. Gamelin, J. Am. Chem. Soc. 125, 13205 (2003).

${ }^{19}$ J. W. P. Hsu, D. R. Tallant, R. L. Simpson, N. A. Missert, and R. G. Copeland, Appl. Phys. Lett. 88, 3 (2006).

${ }^{20}$ F. A. Kro"ger, Chemistry of Imperfect Crystals (North-Holland, Amsterdam, 1964).

${ }^{21}$ F. A. Kroger and H. J. Vink, Solid State Phys., Adv. Res. Appl. 3, 307 (1956).

${ }^{22}$ Y. J. Lin, C. L. Tsai, Y. M. Lu, and C. J. Liu, J. Appl. Phys. 99, 093501 (2006).

${ }^{23}$ S. L. Shi, G. Q. Li, S. J. Xu, Y. Zhao, and G. H. Chen, J. Phys. Chem. B 110, 10475 (2006). 